\title{
Intrathoracic Paravertebral Paraganglioma
}

National Cancer Institute

\section{Source}

National Cancer Institute. Intrathoracic Paravertebral Paraganglioma. NCI Thesaurus.

Code C6411.

A benign or malignant intrathoracic sympathetic paraganglioma arising from

paravertebral paragang lia. In functional tumors, the hypersecretion of catecholamines results in hypertension. 